\title{
Certain sufficient conditions for starlikeness and convexity using a multiplier transformation
}

\author{
Richa Brar and Sukhwinder Singh Billing
}

\begin{abstract}
In the present paper, we study a differential subordination involving a multiplier transformation. Selecting different dominants to our main result, we obtain certain sufficient conditions for starlikeness and convexity of analytic functions. In particular, we obtain the sufficient conditions for parabolic starlikeness and uniform convexity. Some known results appear as particular cases of our main result.
\end{abstract}

Mathematics Subject Classification (2010): 30C80, 30C45.

Keywords: Analytic function, parabolic starlike function, uniformly convex function, starlike function, convex function, differential subordination, multiplier transformation.

\section{Introduction}

Let $\mathcal{A}_{p}$ denote the class of functions of the form

$$
f(z)=z^{p}+\sum_{k=p+1}^{\infty} a_{k} z^{k},(p \in \mathbb{N} ; z \in \mathbb{E})
$$

which are analytic and p-valent in the open unit disk $\mathbb{E}=\{z:|z|<1\}$. Obviously, $\mathcal{A}_{1}=\mathcal{A}$, the class of all analytic functions $f$, normalized by the conditions $f(0)=$ $f^{\prime}(0)-1=0$. Let the functions $f$ and $g$ be analytic in $\mathbb{E}$. We say that $f$ is subordinate to $g$ in $\mathbb{E}$ (written as $f \prec g$ ), if there exists a Schwarz function $\phi$ in $\mathbb{E}$ (i.e. $\phi$ is regular in $|z|<1, \phi(0)=0$ and $|\phi(z)|<1$ for all $z \in \mathbb{E})$ such that

$$
f(z)=g(\phi(z)),|z|<1 \text {. }
$$

Let $\Phi: \mathbb{C}^{2} \times \mathbb{E} \rightarrow \mathbb{C}$ be an analytic function, $p$ an analytic function in $\mathbb{E}$ with $\left(p(z), z p^{\prime}(z) ; z\right) \in \mathbb{C}^{2} \times \mathbb{E}$ for all $z \in \mathbb{E}$ and $h$ be univalent in $\mathbb{E}$. Then the function $p$ is said to satisfy first order differential subordination if

$$
\Phi\left(p(z), z p^{\prime}(z) ; z\right) \prec h(z), \Phi(p(0), 0 ; 0)=h(0) .
$$


A univalent function $q$ is called a dominant of the differential subordination (1.1) if $p(0)=q(0)$ and $p(z) \prec q(z)$ for all $p$ satisfying (1.1). A dominant $\tilde{q}$ that satisfies $\tilde{q}(z) \prec q(z)$ for all dominants $q$ of (1.1), is said to be the best dominant of (1.1). The best dominant is unique up to a rotation of $\mathbb{E}$.

A function $f \in \mathcal{A}_{p}$ is said to be p-valent starlike of order $\alpha(0 \leq \alpha<p)$ in $\mathbb{E}$ if

$$
\Re\left(\frac{z f^{\prime}(z)}{f(z)}\right)>\alpha, z \in \mathbb{E} .
$$

Let $\mathcal{S}_{p}^{*}(\alpha)$ denote the class of p-valent starlike functions of order $\alpha$. Write $\mathcal{S}_{p}^{*}(0)=\mathcal{S}_{p}^{*}$, the class of $\mathrm{p}$-valent starlike functions.

A function $f \in \mathcal{A}_{p}$ is said to be p-valent convex of order $\alpha(0 \leq \alpha<p)$ in $\mathbb{E}$ if it satisfies the condition

$$
\Re\left(1+\frac{z f^{\prime \prime}(z)}{f^{\prime}(z)}\right)>\alpha, z \in \mathbb{E} .
$$

Let the class of such functions be denoted by $\mathcal{K}_{p}(\alpha)$. Let $\mathcal{K}_{p}(0)=\mathcal{K}_{p}$, the class of p-valent convex functions.

A function $f \in \mathcal{A}_{p}$ is said to be p-valent parabolic starlike in $\mathbb{E}$ if

$$
\Re\left(\frac{z f^{\prime}(z)}{f(z)}\right)>\left|\frac{z f^{\prime}(z)}{f(z)}-p\right|, z \in \mathbb{E} .
$$

Let $\mathcal{S}_{\mathcal{P}}^{p}$ denote the class of p-valent parabolic starlike functions. Write $\mathcal{S}_{\mathcal{P}}^{1}=\mathcal{S}_{\mathcal{P}}$, the class of parabolic starlike functions.

A function $f \in \mathcal{A}_{p}$ is said to be uniformly p-valent convex in $\mathbb{E}$ if

$$
\Re\left(1+\frac{z f^{\prime \prime}(z)}{f^{\prime}(z)}\right)>\left|\frac{z f^{\prime \prime}(z)}{f^{\prime}(z)}-(p-1)\right|, z \in \mathbb{E},
$$

and is denoted by $U C V_{p}$, the class of uniformly p-valent convex functions and let $U C V_{1}=U C V$, the class of uniformly convex functions.

Define the parabolic domain $\Omega$ as under

$$
\Omega=\left\{u+i v: u>\sqrt{(u-p)^{2}+v^{2}}\right\} .
$$

Define the function

$$
q(z)=p+\frac{2 p}{\pi^{2}}\left(\log \left(\frac{1+\sqrt{z}}{1-\sqrt{z}}\right)\right)^{2}
$$

by considering only the principal values of logarithmic function. Clearly $q$ maps the unit disk $\mathbb{E}$ onto the domain $\Omega$. Hence the conditions (1.2) and (1.3) are equivalent to

$$
\frac{z f^{\prime}(z)}{f(z)} \prec q(z)
$$

and

$$
1+\frac{z f^{\prime \prime}(z)}{f^{\prime}(z)} \prec q(z),
$$

respectively, where $q$ is given by (1.4).

Ronning [16] and Ma and Minda [12] studied the domain $\Omega$ and the above function 
$q$ in a special case where $p=1$. For $f \in \mathcal{A}_{p}$, we define the multiplier transformation $I_{p}(n, \lambda)$ as

$$
I_{p}(n, \lambda)[f](z)=z^{p}+\sum_{k=p+1}^{\infty}\left(\frac{k+\lambda}{p+\lambda}\right)^{n} a_{k} z^{k}, \text { where } \lambda \geq 0, n \in \mathbb{Z} .
$$

Recently, Billing [2, 3, 4, 5, 6], Singh et al. [18, 19], Brar and Billing [7] investigated the operator $I_{p}(n, \lambda)$ and obtained certain sufficient conditions for starlike and convex functions. Earlier, this operator was studied by Aghalary et al. [1]. In 2003, Cho and Srivastava [9] and Cho and Kim [8] investigated the operator $I_{1}(n, \lambda)$, whereas Uralegaddi and Somanatha [20] studied the operator $I_{1}(n, 1)$. The operator $I_{1}(n, 0)$ is the well-known Sălăgean [17] derivative operator

$$
D^{n} f(z)=z+\sum_{k=2}^{\infty} k^{n} a_{k} z^{k}, n \in \mathbb{N}_{0}=\mathbb{N} \cup\{0\}
$$

and $f \in \mathcal{A}$.

Let $\mathcal{S}_{n}(\alpha)$ denote the class of functions $f \in \mathcal{A}$ for which

$$
\Re\left(\frac{D^{n+1}[f](z)}{D^{n}[f](z)}\right)>\alpha, z \in \mathbb{E}, 0 \leq \alpha<1 .
$$

In 1989, Owa, Shen and Obradović [15] studied this class and obtained some sufficient conditions for $f \in \mathcal{A}$ to be a member of the class $\mathcal{S}_{n}(\alpha)$ in terms of differential inequality. Later on, Li and Owa [11] extended the result of Obradović .

Let $\mathcal{S}_{n}(p, \lambda, \alpha)$ denote the class of functions $f \in \mathcal{A}_{p}$ for which

$$
\Re\left(\frac{I_{p}(n+1, \lambda)[f](z)}{I_{p}(n, \lambda)[f](z)}\right)>\frac{\alpha}{p}, z \in \mathbb{E}, 0 \leq \alpha<p .
$$

In 2008, Billing et al. [18] investigated the above class and proved the sufficient condition for a multivalent function to be a member of this class.

In the present paper, we study a differential subordination involving multiplier transformation $I_{p}(n, \lambda)$ defined above. In particular cases to our main result, we obtain sufficient conditions for parabolic starlikeness, starlikeness, uniform convexity and convexity of multivalent/univalent analytic functions. Some known results are also obtained as particular cases of our main result.

To prove our main results, we shall use the following lemma of Miller and Mocanu ([13], [14], p.132).

Lemma 1.1. Let $q$ be a univalent in $\mathbb{E}$ and let $\theta$ and $\phi$ be analytic in a domain $\mathbb{D}$ containing $q(\mathbb{E})$, with $\phi(w) \neq 0$, when $w \in q(\mathbb{E})$. Set

$$
Q(z)=z q^{\prime}(z) \phi[q(z)], h(z)=\theta[q(z)]+Q(z)
$$

and suppose that either

(i) $h$ is convex, or

(ii) $Q$ is starlike.

In addition, assume that 
(iii) $\Re\left(\frac{z h^{\prime}(z)}{Q(z)}\right)>0$ for all $z$ in $\mathbb{E}$.

If $p$ is analytic in $\mathbb{E}$, with $p(0)=q(0), p(\mathbb{E}) \subset \mathbb{D}$ and

$$
\theta[p(z)]+z p^{\prime}(z) \phi[p(z)] \prec \theta[q(z)]+z q^{\prime}(z) \phi[q(z)],
$$

then $p(z) \prec q(z)$ and $q$ is the best dominant.

\section{Main results}

Theorem 2.1. Let $q$ be a univalent function in $\mathbb{E}$ such that

(i) $\Re\left[1+\frac{z q^{\prime \prime}(z)}{q^{\prime}(z)}-\frac{z q^{\prime}(z)}{q(z)}\right]>0$

(ii) $\Re\left[1+\frac{z q^{\prime \prime}(z)}{q^{\prime}(z)}-\frac{z q^{\prime}(z)}{q(z)}+\frac{p+\lambda}{\alpha} q(z)\right]>0$.

If $f \in \mathcal{A}_{p}$ satisfies

$$
(1-\alpha)\left(\frac{I_{p}(n+1, \lambda)[f](z)}{I_{p}(n, \lambda)[f](z)}\right)+\alpha\left(\frac{I_{p}(n+2, \lambda)[f](z)}{I_{p}(n+1, \lambda)[f](z)}\right) \prec q(z)+\frac{\alpha}{p+\lambda} \frac{z q^{\prime}(z)}{q(z)}
$$

then

$$
\frac{I_{p}(n+1, \lambda)[f](z)}{I_{p}(n, \lambda)[f](z)} \prec q(z),
$$

where $\lambda \geq 0, n \in \mathbb{N}_{0}$ and $\alpha$ is a non zero complex number

Proof. On writing $\frac{I_{p}(n+1, \lambda)[f](z)}{I_{p}(n, \lambda)[f](z)}=u(z)$, in (2.1), we obtain:

$$
u(z)+\frac{\alpha}{p+\lambda} \frac{z u^{\prime}(z)}{u(z)} \prec q(z)+\frac{\alpha}{p+\lambda} \frac{z q^{\prime}(z)}{q(z)},
$$

Let us define the functions $\theta$ and $\phi$ as follows:

$$
\theta(w)=w
$$

and

Therefore,

$$
\phi(w)=\frac{\alpha}{p+\lambda} \frac{1}{w}
$$

and

$$
Q(z)=\phi(q(z)) z q^{\prime}(z)=\frac{\alpha}{p+\lambda} \frac{z q^{\prime}(z)}{q(z)}
$$

$$
h(z)=\theta(q(z))+Q(z)=q(z)+\frac{\alpha}{p+\lambda} \frac{z q^{\prime}(z)}{q(z)} .
$$

On differentiating, we obtain $\frac{z Q^{\prime}(z)}{Q(z)}=1+\frac{z q^{\prime \prime}(z)}{q^{\prime}(z)}-\frac{z q^{\prime}(z)}{q(z)}$ and

$$
\frac{z h^{\prime}(z)}{Q(z)}=1+\frac{z q^{\prime \prime}(z)}{q^{\prime}(z)}-\frac{z q^{\prime}(z)}{q(z)}+\frac{p+\lambda}{\alpha} q(z) .
$$


In view of the given conditions, we see that $\mathrm{Q}$ is starlike and $\Re\left(\frac{z h^{\prime}(z)}{Q(z)}\right)>0$.

Therefore, the proof, now follows from Lemma 1.1.

\section{Special cases and applications}

Setting $\lambda=0$ and $p=1$ in Theorem 2.1, we obtain the following result.

Corollary 3.1. If $f \in \mathcal{A}$ satisfies

$$
(1-\alpha)\left(\frac{D^{n+1}[f](z)}{D^{n}[f](z)}\right)+\alpha\left(\frac{D^{n+2}[f](z)}{D^{n+1}[f](z)}\right) \prec q(z)+\alpha \frac{z q^{\prime}(z)}{q(z)}
$$

then

$$
\frac{D^{n+1}[f](z)}{D^{n}[f](z)} \prec q(z),
$$

where $n \in \mathbb{N}_{0}$ and $\alpha$ is a non zero complex number.

Setting $\alpha=1$ in Theorem 2.1, we get the following result of Shivaprasad Kumar et. al. [10]:

Corollary 3.2. Let $\psi$ be univalent in $\mathbb{E}, \psi(0)=1, \Re \psi(z)>0$ and $\frac{z \psi^{\prime}(z)}{\psi(z)}$ be starlike in $\mathbb{E}$. Suppose $f \in \mathcal{A}_{p}$ satisfies

$$
\frac{I_{p}(n+2, \lambda)[f](z)}{I_{p}(n+1, \lambda)[f](z)} \prec \psi(z)+\frac{z \psi^{\prime}(z)}{(p+\lambda) \psi(z)},
$$

then

$$
\frac{I_{p}(n+1, \lambda)[f](z)}{I_{p}(n, \lambda)[f](z)} \prec \psi(z) .
$$

When we select the dominant $q(z)=1+\frac{2}{\pi^{2}}\left(\log \left(\frac{1+\sqrt{z}}{1-\sqrt{z}}\right)\right)^{2}$ in Theorem 2.1, a simple calculation yields that

$$
\begin{aligned}
1+\frac{z q^{\prime \prime}(z)}{q^{\prime}(z)}-\frac{z q^{\prime}(z)}{q(z)}= & \frac{1+z}{2(1-z)}+\frac{\sqrt{z}}{(1-z) \log \left(\frac{1+\sqrt{z}}{1-\sqrt{z}}\right)}-\frac{\frac{4 \sqrt{z}}{\pi^{2}(1-z)} \log \left(\frac{1+\sqrt{z}}{1-\sqrt{z}}\right)}{1+\frac{2}{\pi^{2}}\left(\log \left(\frac{1+\sqrt{z}}{1-\sqrt{z}}\right)\right)^{2}} \\
& 1+\frac{z q^{\prime \prime}(z)}{q^{\prime}(z)}-\frac{z q^{\prime}(z)}{q(z)}+\frac{p+\lambda}{\alpha} q(z) \\
= & \frac{1+z}{2(1-z)}+\frac{\sqrt{z}}{(1-z) \log \left(\frac{1+\sqrt{z}}{1-\sqrt{z}}\right)}-\frac{\frac{4 \sqrt{z}}{\pi^{2}(1-z)} \log \left(\frac{1+\sqrt{z}}{1-\sqrt{z}}\right)}{1+\frac{2}{\pi^{2}}\left(\log \left(\frac{1+\sqrt{z}}{1-\sqrt{z}}\right)\right)^{2}} \\
+ & \frac{p+\lambda}{\alpha}\left[1+\frac{2}{\pi^{2}}\left(\log \left(\frac{1+\sqrt{z}}{1-\sqrt{z}}\right)\right)^{2}\right] .
\end{aligned}
$$

Thus for a positive real number $\alpha$ we notice that q satisfies the conditions (i) and (ii) of Theorem 2.1. Therefore, we immediately arrive at the following result. 
Corollary 3.3. Let $\alpha$ be a positive real number. If $f \in \mathcal{A}_{p}$ satisfies

$$
\begin{gathered}
(1-\alpha)\left(\frac{I_{p}(n+1, \lambda)[f](z)}{I_{p}(n, \lambda)[f](z)}\right)+\alpha\left(\frac{I_{p}(n+2, \lambda)[f](z)}{I_{p}(n+1, \lambda)[f](z)}\right) \prec 1+\frac{2}{\pi^{2}}\left(\log \left(\frac{1+\sqrt{z}}{1-\sqrt{z}}\right)\right)^{2} \\
+\frac{\alpha}{p+\lambda}\left\{\frac{\frac{4 \sqrt{z}}{\pi^{2}(1-z)} \log \left(\frac{1+\sqrt{z}}{1-\sqrt{z}}\right)}{1+\frac{2}{\pi^{2}}\left(\log \left(\frac{1+\sqrt{z}}{1-\sqrt{z}}\right)\right)^{2}}\right\}
\end{gathered}
$$

then

where $\lambda \geq 0, n \in \mathbb{N}_{0}$.

$$
\frac{I_{p}(n+1, \lambda)[f](z)}{I_{p}(n, \lambda)[f](z)} \prec 1+\frac{2}{\pi^{2}}\left(\log \left(\frac{1+\sqrt{z}}{1-\sqrt{z}}\right)\right)^{2},
$$

Setting $\lambda=n=0$ in Corollary 3.3, we have the following result.

Example 3.4. Let $\alpha$ be a positive real number. If $f \in \mathcal{A}_{p}$ satisfies

$$
\begin{aligned}
(1-\alpha)\left(\frac{z f^{\prime}(z)}{f(z)}\right)+\alpha\left(1+\frac{z f^{\prime \prime}(z)}{f^{\prime}(z)}\right) \prec p & +\frac{2 p}{\pi^{2}}\left(\log \left(\frac{1+\sqrt{z}}{1-\sqrt{z}}\right)\right)^{2} \\
& +\frac{\frac{4 \alpha \sqrt{z}}{\pi^{2}(1-z)} \log \left(\frac{1+\sqrt{z}}{1-\sqrt{z}}\right)}{1+\frac{2}{\pi^{2}}\left(\log \left(\frac{1+\sqrt{z}}{1-\sqrt{z}}\right)\right)^{2}},
\end{aligned}
$$

then $f \in \mathcal{S}_{\mathcal{P}}^{p}$.

Setting $p=1$ in above example, we get the following result of Brar and Billing [7]:

Example 3.5. Let $\alpha$ be a positive real number. If $f \in \mathcal{A}$ satisfies

then $f \in \mathcal{S}_{\mathcal{P}}$.

$$
\begin{aligned}
(1-\alpha)\left(\frac{z f^{\prime}(z)}{f(z)}\right)+\alpha\left(1+\frac{z f^{\prime \prime}(z)}{f^{\prime}(z)}\right) \prec 1 & +\frac{2}{\pi^{2}}\left(\log \left(\frac{1+\sqrt{z}}{1-\sqrt{z}}\right)\right)^{2} \\
& +\frac{\frac{4 \alpha \sqrt{z}}{\pi^{2}(1-z)} \log \left(\frac{1+\sqrt{z}}{1-\sqrt{z}}\right)}{1+\frac{2}{\pi^{2}}\left(\log \left(\frac{1+\sqrt{z}}{1-\sqrt{z}}\right)\right)^{2}},
\end{aligned}
$$

Setting $\lambda=0, n=1$ in Corollary 3.3, we obtain:

Example 3.6. Let $\alpha$ be a positive real number. If $f \in \mathcal{A}_{p}$ satisfies

$$
\begin{aligned}
& (1-\alpha)\left(1+\frac{z f^{\prime \prime}(z)}{f^{\prime}(z)}\right)+\alpha\left(1+\frac{2 z f^{\prime \prime}(z)+z^{2} f^{\prime \prime \prime}(z)}{f^{\prime}(z)+z f^{\prime \prime}(z)}\right) \\
& \prec p+\frac{2 p}{\pi^{2}}\left(\log \left(\frac{1+\sqrt{z}}{1-\sqrt{z}}\right)\right)^{2}+\frac{\frac{4 \alpha \sqrt{z}}{\pi^{2}(1-z)} \log \left(\frac{1+\sqrt{z}}{1-\sqrt{z}}\right)}{1+\frac{2}{\pi^{2}}\left(\log \left(\frac{1+\sqrt{z}}{1-\sqrt{z}}\right)\right)^{2}},
\end{aligned}
$$

then $f \in U C V_{p}$.

Setting $p=1$ in above example, we get: 
Example 3.7. Let $\alpha$ be a positive real number. If $f \in \mathcal{A}$ satisfies

$$
\begin{gathered}
(1-\alpha)\left(1+\frac{z f^{\prime \prime}(z)}{f^{\prime}(z)}\right)+\alpha\left(1+\frac{2 z f^{\prime \prime}(z)+z^{2} f^{\prime \prime \prime}(z)}{f^{\prime}(z)+z f^{\prime \prime}(z)}\right) \prec 1+\frac{2}{\pi^{2}}\left(\log \left(\frac{1+\sqrt{z}}{1-\sqrt{z}}\right)\right)^{2} \\
+\frac{\frac{4 \alpha \sqrt{z}}{\pi^{2}(1-z)} \log \left(\frac{1+\sqrt{z}}{1-\sqrt{z}}\right)}{1+\frac{2}{\pi^{2}}\left(\log \left(\frac{1+\sqrt{z}}{1-\sqrt{z}}\right)\right)^{2}},
\end{gathered}
$$

then $f \in U C V$.

When we select the dominant $q(z)=\frac{1+(1-2 \beta) z}{1-z}, 0 \leq \beta<1$ in Theorem 2.1, an easy calculation yields that

$$
\begin{gathered}
1+\frac{z q^{\prime \prime}(z)}{q^{\prime}(z)}-\frac{z q^{\prime}(z)}{q(z)}=\frac{1+(1-2 \beta) z^{2}}{(1-z)(1+(1-2 \beta) z)}, \\
1+\frac{z q^{\prime \prime}(z)}{q^{\prime}(z)}-\frac{z q^{\prime}(z)}{q(z)}+\frac{p+\lambda}{\alpha} q(z)=\frac{1+(1-2 \beta) z^{2}}{(1-z)(1+(1-2 \beta) z)}+\frac{p+\lambda}{\alpha}\left(\frac{1+(1-2 \beta) z}{1-z}\right) .
\end{gathered}
$$

Thus for a positive real number $\alpha$ we notice that q satisfies the conditions (i) and (ii) of Theorem 2.1. Therefore, we get the following result.

Corollary 3.8. Let $\alpha$ be a positive real number. If $f \in \mathcal{A}_{p}$ satisfies

$$
\begin{gathered}
(1-\alpha)\left(\frac{I_{p}(n+1, \lambda)[f](z)}{I_{p}(n, \lambda)[f](z)}\right)+\alpha\left(\frac{I_{p}(n+2, \lambda)[f](z)}{I_{p}(n+1, \lambda)[f](z)}\right) \prec \frac{1+(1-2 \beta) z}{1-z} \\
+\frac{2 \alpha(1-\beta) z}{(p+\lambda)(1-z)(1+(1-2 \beta) z)}, 0 \leq \beta<1
\end{gathered}
$$

then

$$
\frac{I_{p}(n+1, \lambda)[f](z)}{I_{p}(n, \lambda)[f](z)} \prec \frac{1+(1-2 \beta) z}{1-z}
$$

where $\lambda \geq 0, n \in \mathbb{N}_{0}$.

Setting $\lambda=n=0$ in Corollary 3.8, we obtain the following criterion for starlikeness.

Example 3.9. Let $\alpha$ be a positive real number. If $f \in \mathcal{A}_{p}$ satisfies

$$
\begin{gathered}
(1-\alpha)\left(\frac{z f^{\prime}(z)}{f(z)}\right)+\alpha\left(1+\frac{z f^{\prime \prime}(z)}{f^{\prime}(z)}\right) \prec \frac{p+p(1-2 \beta) z}{1-z} \\
+\frac{2 \alpha(1-\beta) z}{(1-z)(1+(1-2 \beta) z)}, 0 \leq \beta<1,
\end{gathered}
$$

then

$$
\frac{z f^{\prime}(z)}{f(z)} \prec \frac{p+p(1-2 \beta) z}{1-z} .
$$

The selection $p=1$ in above example, yields the following result: 
Example 3.10. Let $\alpha$ be a positive real number. If $f \in \mathcal{A}$ satisfies

then $f \in \mathcal{S}^{*}(\beta)$.

$$
\begin{gathered}
(1-\alpha)\left(\frac{z f^{\prime}(z)}{f(z)}\right)+\alpha\left(1+\frac{z f^{\prime \prime}(z)}{f^{\prime}(z)}\right) \prec \frac{1+(1-2 \beta) z}{1-z} \\
+\frac{2 \alpha(1-\beta) z}{(1-z)(1+(1-2 \beta) z)}, 0 \leq \beta<1
\end{gathered}
$$

Setting $\lambda=0, n=1$ in Corollary 3.8, we obtain the following result of convexity.

Example 3.11. Let $\alpha$ be a positive real number. If $f \in \mathcal{A}_{p}$ satisfies

$$
\begin{gathered}
(1-\alpha)\left(1+\frac{z f^{\prime \prime}(z)}{f^{\prime}(z)}\right)+\alpha\left(1+\frac{2 z f^{\prime \prime}(z)+z^{2} f^{\prime \prime \prime}(z)}{f^{\prime}(z)+z f^{\prime \prime}(z)}\right) \prec \frac{p+p(1-2 \beta) z}{1-z} \\
+\frac{2 \alpha(1-\beta) z}{(1-z)(1+(1-2 \beta) z)}, 0 \leq \beta<1,
\end{gathered}
$$

then $1+\frac{z f^{\prime \prime}(z)}{f^{\prime}(z)} \prec \frac{p+p(1-2 \beta) z}{1-z}$.

Setting $p=1$ in above example, we obtain:

Example 3.12. Let $\alpha$ be a positive real number. If $f \in \mathcal{A}$ satisfies

then $f \in \mathcal{K}(\beta)$.

$$
\begin{gathered}
(1-\alpha)\left(1+\frac{z f^{\prime \prime}(z)}{f^{\prime}(z)}\right)+\alpha\left(1+\frac{2 z f^{\prime \prime}(z)+z^{2} f^{\prime \prime \prime}(z)}{f^{\prime}(z)+z f^{\prime \prime}(z)}\right) \prec \frac{1+(1-2 \beta) z}{1-z} \\
+\frac{2 \alpha(1-\beta) z}{(1-z)(1+(1-2 \beta) z)}, 0 \leq \beta<1,
\end{gathered}
$$

When we select the dominant $q(z)=e^{z}$ in Theorem 2.1, a simple calculation gives that

$$
\begin{gathered}
1+\frac{z q^{\prime \prime}(z)}{q^{\prime}(z)}-\frac{z q^{\prime}(z)}{q(z)}=1 \\
1+\frac{z q^{\prime \prime}(z)}{q^{\prime}(z)}-\frac{z q^{\prime}(z)}{q(z)}+\frac{p+\lambda}{\alpha} q(z)=1+\frac{p+\lambda}{\alpha} e^{z} .
\end{gathered}
$$

Thus for a positive real number $\alpha$ we notice that q satisfies the conditions (i) and (ii) of Theorem 2.1. We obtain the following result.

Corollary 3.13. Let $\alpha$ be a positive real number. If $f \in \mathcal{A}_{p}$ satisfies

$$
(1-\alpha)\left(\frac{I_{p}(n+1, \lambda)[f](z)}{I_{p}(n, \lambda)[f](z)}\right)+\alpha\left(\frac{I_{p}(n+2, \lambda)[f](z)}{I_{p}(n+1, \lambda)[f](z)}\right) \prec e^{z}+\frac{\alpha z}{p+\lambda},
$$

then

$$
\frac{I_{p}(n+1, \lambda)[f](z)}{I_{p}(n, \lambda)[f](z)} \prec e^{z}
$$

where $\lambda \geq 0, n \in \mathbb{N}_{0}$.

The selection $\lambda=n=0$ in Corollary 3.13, yields the following result. 
Example 3.14. Let $\alpha$ be a positive real number. If $f \in \mathcal{A}_{p}$ satisfies

$$
(1-\alpha)\left(\frac{z f^{\prime}(z)}{f(z)}\right)+\alpha\left(1+\frac{z f^{\prime \prime}(z)}{f^{\prime}(z)}\right) \prec p e^{z}+\alpha z,
$$

then $f \in \mathcal{S}_{p}^{*}$.

Setting $p=1$ in above example, we get:

Example 3.15. Let $\alpha$ be a positive real number. If $f \in \mathcal{A}$ satisfies

$$
(1-\alpha)\left(\frac{z f^{\prime}(z)}{f(z)}\right)+\alpha\left(1+\frac{z f^{\prime \prime}(z)}{f^{\prime}(z)}\right) \prec e^{z}+\alpha z,
$$

then $f \in \mathcal{S}^{*}$.

Setting $\lambda=0, n=1$ in Corollary 3.13, we obtain the following result.

Example 3.16. Let $\alpha$ be a positive real number. If $f \in \mathcal{A}_{p}$ satisfies

$$
(1-\alpha)\left(1+\frac{z f^{\prime \prime}(z)}{f^{\prime}(z)}\right)+\alpha\left(1+\frac{2 z f^{\prime \prime}(z)+z^{2} f^{\prime \prime \prime}(z)}{f^{\prime}(z)+z f^{\prime \prime}(z)}\right) \prec p e^{z}+\alpha z,
$$

then $f \in \mathcal{K}_{p}$.

Setting $p=1$ in above example, we get:

Example 3.17. Let $\alpha$ be a positive real number. If $f \in \mathcal{A}$ satisfies

$$
(1-\alpha)\left\{1+\frac{z f^{\prime \prime}(z)}{f^{\prime}(z)}\right\}+\alpha\left(1+\frac{2 z f^{\prime \prime}(z)+z^{2} f^{\prime \prime \prime}(z)}{f^{\prime}(z)+z f^{\prime \prime}(z)}\right) \prec e^{z}+\alpha z,
$$

then $f \in \mathcal{K}$.

When we select the dominant $q(z)=\frac{\alpha^{\prime}(1-z)}{\alpha^{\prime}-z}, \alpha^{\prime}>1$ in Theorem 2.1, a simple calculation yields that

$$
\begin{gathered}
1+\frac{z q^{\prime \prime}(z)}{q^{\prime}(z)}-\frac{z q^{\prime}(z)}{q(z)}=\frac{1}{1-z}+\frac{z}{\alpha^{\prime}-z} \\
1+\frac{z q^{\prime \prime}(z)}{q^{\prime}(z)}-\frac{z q^{\prime}(z)}{q(z)}+\frac{p+\lambda}{\alpha} q(z)=\frac{1}{1-z}+\frac{z}{\alpha^{\prime}-z}+\frac{p+\lambda}{\alpha}\left(\frac{\alpha^{\prime}(1-z)}{\alpha^{\prime}-z}\right) .
\end{gathered}
$$

Thus for a positive real number $\alpha$ we notice that q satisfies the conditions (i) and (ii) of Theorem 2.1. Therefore, we, immediately arrive at the following result.

Corollary 3.18. Let $\alpha$ be a positive real number. If $f \in \mathcal{A}_{p}$ satisfies

then

$$
\begin{aligned}
(1-\alpha)\left(\frac{I_{p}(n+1, \lambda)[f](z)}{I_{p}(n, \lambda)[f](z)}\right)+\alpha\left(\frac{I_{p}(n+2, \lambda)[f](z)}{I_{p}(n+1, \lambda)[f](z)}\right) \\
\prec \frac{\alpha^{\prime}(1-z)}{\alpha^{\prime}-z}+\frac{\alpha z\left(1-\alpha^{\prime}\right)}{(p+\lambda)(1-z)\left(\alpha^{\prime}-z\right)}, \alpha^{\prime}>1,
\end{aligned}
$$

where $\lambda \geq 0, n \in \mathbb{N}_{0}$.

$$
\frac{I_{p}(n+1, \lambda)[f](z)}{I_{p}(n, \lambda)[f](z)} \prec \frac{\alpha^{\prime}(1-z)}{\alpha^{\prime}-z},
$$


Setting $\lambda=n=0$ in Corollary 3.18, we obtain the following result for starlikeness.

Example 3.19. Let $\alpha$ be a positive real number. If $f \in \mathcal{A}_{p}$ satisfies

$$
(1-\alpha)\left(\frac{z f^{\prime}(z)}{f(z)}\right)+\alpha\left(1+\frac{z f^{\prime \prime}(z)}{f^{\prime}(z)}\right) \prec \frac{p \alpha^{\prime}(1-z)}{\alpha^{\prime}-z}+\frac{\alpha z\left(1-\alpha^{\prime}\right)}{(1-z)\left(\alpha^{\prime}-z\right)}, \alpha^{\prime}>1,
$$

then $f \in \mathcal{S}_{p}^{*}$.

Setting $p=1$ in above example, we have:

Example 3.20. Let $\alpha$ be a positive real number. If $f \in \mathcal{A}$ satisfies

$$
(1-\alpha)\left(\frac{z f^{\prime}(z)}{f(z)}\right)+\alpha\left(1+\frac{z f^{\prime \prime}(z)}{f^{\prime}(z)}\right) \prec \frac{\alpha^{\prime}(1-z)}{\alpha^{\prime}-z}+\frac{\alpha z\left(1-\alpha^{\prime}\right)}{(1-z)\left(\alpha^{\prime}-z\right)}, \alpha^{\prime}>1,
$$

then $f \in \mathcal{S}^{*}$.

Setting $\lambda=0, n=1$ in Corollary 3.18, we obtain the following result.

Example 3.21. Let $\alpha$ be a positive real number. If $f \in \mathcal{A}_{p}$ satisfies

$$
\begin{gathered}
(1-\alpha)\left(1+\frac{z f^{\prime \prime}(z)}{f^{\prime}(z)}\right)+\alpha\left(1+\frac{2 z f^{\prime \prime}(z)+z^{2} f^{\prime \prime \prime}(z)}{f^{\prime}(z)+z f^{\prime \prime}(z)}\right) \\
\prec \frac{p \alpha^{\prime}(1-z)}{\alpha^{\prime}-z}+\frac{\alpha z\left(1-\alpha^{\prime}\right)}{(1-z)\left(\alpha^{\prime}-z\right)}, \alpha^{\prime}>1,
\end{gathered}
$$

then $f \in \mathcal{K}_{p}$.

Setting $p=1$ in above example, we get:

Example 3.22. Let $\alpha$ be a positive real number. If $f \in \mathcal{A}$ satisfies

$$
\begin{array}{r}
(1-\alpha)\left(1+\frac{z f^{\prime \prime}(z)}{f^{\prime}(z)}\right)+\alpha\left(1+\frac{2 z f^{\prime \prime}(z)+z^{2} f^{\prime \prime \prime}(z)}{f^{\prime}(z)+z f^{\prime \prime}(z)}\right) \\
\prec \frac{\alpha^{\prime}(1-z)}{\alpha^{\prime}-z}+\frac{\alpha z\left(1-\alpha^{\prime}\right)}{(1-z)\left(\alpha^{\prime}-z\right)}, \alpha^{\prime}>1,
\end{array}
$$

then $f \in \mathcal{K}$.

When we select the dominant $q(z)=\left(\frac{1+z}{1-z}\right)^{\gamma}, 0<\gamma \leq 1$ such that $q(0)=1$ in Theorem 2.1, an easy calculation yields that

$$
\begin{gathered}
1+\frac{z q^{\prime \prime}(z)}{q^{\prime}(z)}-\frac{z q^{\prime}(z)}{q(z)}=\frac{1+z^{2}}{1-z^{2}} \\
1+\frac{z q^{\prime \prime}(z)}{q^{\prime}(z)}-\frac{z q^{\prime}(z)}{q(z)}+\frac{(p+\lambda)}{\alpha} q(z)=\frac{1+z^{2}}{1-z^{2}}+\frac{(p+\lambda)}{\alpha}\left(\frac{1+z}{1-z}\right)^{\gamma} .
\end{gathered}
$$

Thus for a positive real number $\alpha$ we notice that q satisfies the conditions (i) and (ii) of Theorem 2.1. Therefore we obtain the following result. 
Corollary 3.23. Let $\alpha$ be a positive real number. If $f \in \mathcal{A}_{p}$ satisfies

then

$$
\begin{array}{r}
(1-\alpha)\left(\frac{I_{p}(n+1, \lambda)[f](z)}{I_{p}(n, \lambda)[f](z)}\right)+\alpha\left(\frac{I_{p}(n+2, \lambda)[f](z)}{I_{p}(n+1, \lambda)[f](z)}\right) \\
\prec \frac{(1+z)^{\gamma}}{(1-z)^{\gamma}}+\frac{2 \alpha \gamma z}{(p+\lambda)\left(1-z^{2}\right)}, 0<\gamma \leq 1,
\end{array}
$$

where $\lambda \geq 0, n \in \mathbb{N}_{0}$.

$$
\frac{I_{p}(n+1, \lambda)[f](z)}{I_{p}(n, \lambda)[f](z)} \prec\left(\frac{1+z}{1-z}\right)^{\gamma}
$$

Setting $\lambda=n=0$ in Corollary 3.23, we get the following result.

Example 3.24. Let $\alpha$ be a positive real number. If $f \in \mathcal{A}_{p}$ satisfies

$$
(1-\alpha)\left(\frac{z f^{\prime}(z)}{f(z)}\right)+\alpha\left(1+\frac{z f^{\prime \prime}(z)}{f^{\prime}(z)}\right) \prec p \frac{(1+z)^{\gamma}}{(1-z)^{\gamma}}+\frac{2 \alpha \gamma z}{\left(1-z^{2}\right)}, 0<\gamma \leq 1,
$$

then

$$
\frac{z f^{\prime}(z)}{f(z)} \prec p\left(\frac{1+z}{1-z}\right)^{\gamma}
$$

Setting $p=1$ in above example, we get:

Example 3.25. Let $\alpha$ be a positive real number. If $f \in \mathcal{A}$ satisfies

$$
(1-\alpha)\left(\frac{z f^{\prime}(z)}{f(z)}\right)+\alpha\left(1+\frac{z f^{\prime \prime}(z)}{f^{\prime}(z)}\right) \prec \frac{(1+z)^{\gamma}}{(1-z)^{\gamma}}+\frac{2 \alpha \gamma z}{1-z^{2}}, 0<\gamma \leq 1,
$$

then

$$
\frac{z f^{\prime}(z)}{f(z)} \prec\left(\frac{1+z}{1-z}\right)^{\gamma}
$$

Setting $\lambda=0, n=1$ in Corollary 3.23, we obtain the following result.

Example 3.26. Let $\alpha$ be a positive real number. If $f \in \mathcal{A}_{p}$ satisfies

then

$$
\begin{gathered}
(1-\alpha)\left(1+\frac{z f^{\prime \prime}(z)}{f^{\prime}(z)}\right)+\alpha\left(1+\frac{2 z f^{\prime \prime}(z)+z^{2} f^{\prime \prime \prime}(z)}{f^{\prime}(z)+z f^{\prime \prime}(z)}\right) \\
\prec \frac{p(1+z)^{\gamma}}{(1-z)^{\gamma}}+\frac{2 \alpha \gamma z}{\left(1-z^{2}\right)}, 0<\gamma \leq 1,
\end{gathered}
$$

$$
1+\frac{z f^{\prime \prime}(z)}{f^{\prime}(z)} \prec p\left(\frac{1+z}{1-z}\right)^{\gamma} .
$$

Setting $p=1$ in above example, we get:

Example 3.27. Let $\alpha$ be a positive real number. If $f \in \mathcal{A}$ satisfies

$$
(1-\alpha)\left(1+\frac{z f^{\prime \prime}(z)}{f^{\prime}(z)}\right)+\alpha\left(1+\frac{2 z f^{\prime \prime}(z)+z^{2} f^{\prime \prime \prime}(z)}{f^{\prime}(z)+z f^{\prime \prime}(z)}\right) \prec \frac{(1+z)^{\gamma}}{(1-z)^{\gamma}}+\frac{2 \alpha \gamma z}{1-z^{2}}, 0<\gamma \leq 1,
$$

then

$$
1+\frac{z f^{\prime \prime}(z)}{f^{\prime}(z)} \prec\left(\frac{1+z}{1-z}\right)^{\gamma}
$$


When we select the dominant $q(z)=1+a z ; 0 \leq a<1$ in Theorem 2.1, a simple calculation yields that

$$
\begin{gathered}
1+\frac{z q^{\prime \prime}(z)}{q^{\prime}(z)}-\frac{z q^{\prime}(z)}{q(z)}=\frac{1}{1+a z} \\
1+\frac{z q^{\prime \prime}(z)}{q^{\prime}(z)}-\frac{z q^{\prime}(z)}{q(z)}+\frac{p+\lambda}{\alpha} q(z)=\frac{1}{1+a z}+\frac{p+\lambda}{\alpha}(1+a z) .
\end{gathered}
$$

Thus for a positive real number $\alpha$ we notice that q satisfies the conditions (i) and (ii) of Theorem 2.1. Therefore, we immediately deduce the following result.

Corollary 3.28. Let $\alpha$ be a positive real number. If $f \in \mathcal{A}_{p}$ satisfies

$$
\begin{array}{r}
(1-\alpha)\left(\frac{I_{p}(n+1, \lambda)[f](z)}{I_{p}(n, \lambda)[f](z)}\right)+\alpha\left(\frac{I_{p}(n+2, \lambda)[f](z)}{I_{p}(n+1, \lambda)[f](z)}\right) \\
\prec 1+a z+\frac{\alpha a z}{(p+\lambda)(1+a z)}, 0 \leq a<1,
\end{array}
$$

then

$$
\frac{I_{p}(n+1, \lambda)[f](z)}{I_{p}(n, \lambda)[f](z)} \prec 1+a z
$$

where $n \in \mathbb{N}_{0}$.

Setting $\lambda=n=0$ in Corollary 3.28, we get the following result.

Example 3.29. Let $\alpha$ be a positive real number. If $f \in \mathcal{A}_{p}$ satisfies

$$
(1-\alpha)\left(\frac{z f^{\prime}(z)}{f(z)}\right)+\alpha\left(1+\frac{z f^{\prime \prime}(z)}{f^{\prime}(z)}\right) \prec p(1+a z)+\frac{\alpha a z}{1+a z}, 0 \leq a<1,
$$

then $f \in \mathcal{S}_{p}^{*}$.

The substitution $p=1$ in above example, yields the following result:,

Example 3.30. Let $\alpha$ be a positive real number. If $f \in \mathcal{A}$ satisfies

$$
(1-\alpha)\left(\frac{z f^{\prime}(z)}{f(z)}\right)+\alpha\left(1+\frac{z f^{\prime \prime}(z)}{f^{\prime}(z)}\right) \prec 1+a z+\frac{\alpha a z}{1+a z}, 0 \leq a<1,
$$

then $f \in \mathcal{S}^{*}$.

Setting $\lambda=0, n=1$ in Corollary 3.28, we obtain the following result.

Example 3.31. Let $\alpha$ be a positive real number. If $f \in \mathcal{A}_{p}$ satisfies

$$
\begin{gathered}
(1-\alpha)\left(1+\frac{z f^{\prime \prime}(z)}{f^{\prime}(z)}\right)+\alpha\left(1+\frac{2 z f^{\prime \prime}(z)+z^{2} f^{\prime \prime \prime}(z)}{f^{\prime}(z)+z f^{\prime \prime}(z)}\right) \\
\prec p(1+a z)+\frac{\alpha a z}{1+a z}, 0 \leq a<1,
\end{gathered}
$$

then $f \in \mathcal{K}_{p}$.

Setting $p=1$ in above example, we get: 
Example 3.32. Let $\alpha$ be a positive real number. If $f \in \mathcal{A}$ satisfies

$(1-\alpha)\left(1+\frac{z f^{\prime \prime}(z)}{f^{\prime}(z)}\right)+\alpha\left(1+\frac{2 z f^{\prime \prime}(z)+z^{2} f^{\prime \prime \prime}(z)}{f^{\prime}(z)+z f^{\prime \prime}(z)}\right) \prec 1+a z+\frac{\alpha a z}{1+a z}, 0 \leq a<1$, then $f \in \mathcal{K}$.

Acknowledgement. We are thankful to the referee for his valuable suggestions.

\section{References}

[1] Aghalary, R., Ali, R.M., Joshi, S.B., Ravichandran, V., Inequalities for analytic functions defined by certain linear operators, Int. J. Math. Sci., 4(2005), no. 2, 267-274.

[2] Billing, S.S., Certain differential subordination involving a multiplier transformation, Scientia Magna, 8(2012), no. 1, 87-93.

[3] Billing, S.S., Differential inequalities implying starlikeness and convexity, Acta Univ. M. Belii, ser. Math., 20(2012), 3-8.

[4] Billing, S.S., A subordination theorem involving a multiplier transformation, Int. J. Anal. Appl., 1(2013), no. 2, 100-105.

[5] Billing, S.S., A multiplier transformation and conditions for starlike and convex functions, Math. Sci. Res. J., 17(2013), no. 9, 239-244.

[6] Billing, S.S., Differential inequalities and criteria for starlike and convex functions, Stud. Univ. Babeş-Bolyai Math., 59(2014), no. 2, 191-198.

[7] Brar, R., Billing S.S., Certain sufficient conditions for parabolic starlike and uniformly close-to-convex functions, Stud. Univ. Babeş-Bolyai Math., 61(2016), no. 1, 53-62.

[8] Cho, N.E., Kim, T.H., Multiplier transformations and stronlgy close-to-convex functions, Bull. Korean Math. Soc., 40(2003), 399-410.

[9] Cho, N.E., Srivastava, H.M., Argument estimates of certain analytic functions defined by a class of multiplier transformations, Math. Comput. Model., 37(2003), 39-49.

[10] Kumar, S.S., Ravichandran V., Taneja H.C., Classes of multivalent functions defined by Dziok-Srivastava linear operators, Kyungpook Math. J., 46(2006), 97-109.

[11] Li, J., Owa, S., Properties of the sălăgean operator, Georgian Math. J., 5(1998), no. 4, 361-366.

[12] Ma, W.C., Minda, D., Uniformly convex functions, Ann. Polon. Math., 57(1992), no. 2, 165-175.

[13] Miller, S.S., Mocanu, P.T., On some classes of first order differential subordinations, Michigan Math. J., 32(1985), 185-195.

[14] Miller, S.S., Mocanu, P.T., Differential subordination: Theory and applications, Marcel Dekker, New York and Basel, 2000.

[15] Owa, S., Shen, C.Y., Obradović, M., Certain subcalsses of analytic functions, Tamkang J. Math., 20(1989), 105-115.

[16] Ronning, F., Uniformly convex functions and a corresponding class of starlike functions, Proc. Amer. Math. Soc., 118(1993), no. 1, 189-196.

[17] Sălăgean, G.S., Subclasses of univalent functions, Lecture Notes in Math., SpringerVerlag, Heidelberg, 1013(1983), 362-372. 
[18] Singh, S., Gupta, S., Singh, S., On a class of multivalent functions defined by a multiplier transformation, Mat. Vesnik, 60(2008), 87-94.

[19] Singh, S., Gupta, S., Singh, S., On starlikeness and convexity of analytic functions satisfying a differential inequality, J. Inequal. Pure and Appl. Math., 9(2008), no. 3, art. 81, 1-7.

[20] Uralegaddi, B.A., Somanatha, C., Certain classes of univalent functions, Current Topics in Analytic Function Theory, H.M. Srivastava and S. Owa (ed.), World Scientific, Singapore, 1992, 371-374.

Richa Brar

"Sri Guru Granth Sahib World" University

Department of Mathematics

Fatehgarh Sahib, 140407 Punjab, India

e-mail: richabrar4@gmail.com

Sukhwinder Singh Billing

"Sri Guru Granth Sahib World" University

Department of Mathematics

Fatehgarh Sahib, 140407 Punjab, India

e-mail: ssbilling@gmail.com 